“(C) 2015 IEEE. Personal use of this material is permitted. Permission from IEEE must be obtained for all other uses, in any current or future media, including reprinting/republishing this material for advertising or promotional purposes, creating new collective works, for resale or redistribution to servers or lists, or reuse of any copyrighted component of this work in other works." 


\title{
A Survey of Direction of Arrival Estimation Techniques and Implementation of Channel Estimation based on SCME
}

\author{
Shouman Barua, Sinh Cong Lam ${ }^{1}$, Pantha Ghosal, Shiqi Xing ${ }^{1}$, Kumbesan Sandrasegaran
}

School of Computing and Communications, University of Technology, Sydney

\{shouman.barua, Pantha.ghosal, kumbesan.sandrasegaran\}@uts.edu.au, \{SinhCong.Lam, Shiqi.Xing-1 $\}^{1} @$ student.uts.edu.au

\begin{abstract}
In this paper, basics of Direction of Arrival (DOA) estimation techniques were reviewed along with simulated results. It justifies the pros and cons of each technique. The purposes of using smart antenna system in this paper are regarded with the respect of DOA estimation, Path Delays estimation, and accurate channel estimation between a transmitter and an array of receivers. Considering the uplink transmission system, a source antenna transmits a signal and a group of antennas placed uniformly receives the signal. Then the target was to perform the common DOA estimation techniques to analyze the source signal. Since the Long Term Evolution (LTE) is the currently available standard, this model was used to estimate the channel with the help of a 2D harmonic model. Spatial Channel Model was considered for the simulation and finally, a uniform rectangular array model is compared with a multipath channel equation to estimate the channel.
\end{abstract}

Index Terms-DOA, LTE, Channel Estimation, ESPRIT, URA and $U L A$

\section{INTRODUCTION}

Although during the last few decades wireless communication was able to fulfill so many requirements other than just simple communicating between two ends, the stateof- the-art technologies are not still able to meet all the requirements created by the people. The main interest of recent research is to design a model efficiently to estimate the location, direction, distance, and path delay (induced by the fading channel) of the source. A proper channel model plays an important role to perform those tasks efficiently. Therefore, the problem of proper channel estimation arises in a large variety of important applications for the researchers.

To meet the demand of increasing data rate, LTE was standardized by the 3GPP. It is also one of the most popular technologies because it provides low latency, higher spectrum efficiently and seamless mobility. The two main latest technologies i.e., MIMO (Multiple Input Multiple Output) and OFDM (Orthogonal Frequency Division Multiple Access) in wireless communication system are incorporated in LTE system so that it performs better than any earlier technologies.

Only uplink parameters have been considered the uplink to estimate the channel. Proper utilizing and planning for the uplink is very important because the power of user equipment (UE) is limited. Calculation of the DOA and delay can easily be estimated from the estimated channel.
In this paper, the simulation was performed based on SCME (Spatial Channel Model Extended) platform. Channel in wireless communication stands for the link between two end users of the communications system. The performance of a communication system between two ends is greatly determined by the behaviors of the channel characteristics. The channel characteristics determine signal transformation while propagating through the channel between transmitters and receivers. Accurate channel information is crucial for both the transmitter and receiver ends to perform their best services. In order to estimate the channel, LTE reference signal specified by 3GPP, which is characterized by Zadoff-Chu (ZC) sequences were considered.

Next part is dedicated to the basic DOA estimation techniques. In part three, it tries to focus on the theory of DOA estimation using Uniform Rectangular Array (URA) and a related technique namely unitary 2D ESPRIT. An algorithm for estimating the channel has been discussed in part four. Experimental setup and results have been discussed in this part five and finally, the paper is concluded with a summary.

\section{DIRECTION OF ARRIVAL ESTIMATION TECHNIQUES}

A Uniform Linear Array (ULA) consisting of L elements are placed with uniform distance $d$ between two adjacent ones as shown in Figure 1. Assume the total number of sources to be $P$. The wavefront of the far-field signal (at an angle) impinges first on the left most antenna element of the receiving antenna array. Then, each antenna receives the signal with an amount of phase shift that can be calculated with the help of geometry of the uniform linear array.
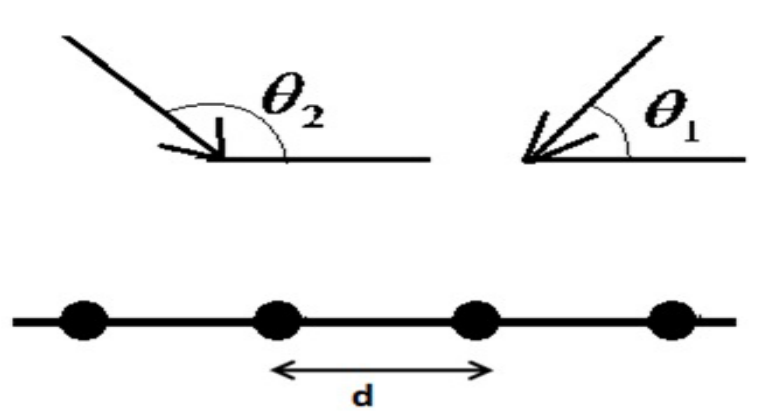

Figure 1: Concept of basic beamforming technique. 
Signal received by the first antenna is $s_{1}(t)=\mathrm{e}^{j 2 \pi f_{0} t}$ where, $f_{o}$ is the frequency and $t$ represents time. Therefore, the received signal by the second antenna is $s_{2}(t)=s_{1}(t-\tau)$ where, $\tau$ is the delayed time $=s_{1}(t) \mathrm{e}^{j \mu}$ where, $\mu_{i}=-\frac{2 \pi}{\lambda} d \cos \theta_{i}$ here, $\lambda$ indicates wavelength. Finally, the signal model equation can be written as follows:

\section{$\mathbf{x}(t)=\mathbf{A}(\theta) \mathbf{s}(t)+\mathbf{n}(t)$}

where, $\mathbf{x}(t)=\left[\begin{array}{llll}x_{l}(t) & x_{2}(t) \ldots x_{L}(t)\end{array}\right]^{\mathrm{T}}$ is the data column vector received by the $L$ array receiving antennas, $\mathbf{s}(t)=\left[s_{1}(t)\right.$ $\left.s_{2}(t) \ldots s_{P}(t)\right]^{\mathrm{T}}$ is the signal column vector generated by $P$ sources, $\mathbf{n}(t)=\left[n_{1}(t) n_{2}(t) \ldots n_{L}(t)\right]^{\mathrm{T}}$ is the zero mean spatially uncorrelated noise, and $\mathbf{A}(\theta)$ is called the $(L \times P)$ steering matrix which is as follows.

$$
\mathbf{A}(\theta)=\left[\begin{array}{ccccc}
1 & \ldots & 1 & \ldots & 1 \\
e^{j \mu_{1}} & \ldots & e^{j \mu_{i}} & \ldots & e^{j \mu_{P}} \\
\vdots & \vdots & \vdots & \vdots & \vdots \\
e^{j(\mathrm{~L}-1) \mu_{1}} & \cdots & e^{j(\mathrm{~L}-1) \mu_{i}} & \cdots & e^{j(\mathrm{~L}-1) \mu_{P}}
\end{array}\right]
$$

Covariance matrix can be defined as

$$
\hat{\mathbf{R}}_{\mathbf{X x}}=\frac{1}{T} \sum_{t=1}^{T} \mathbf{x}(t) \mathbf{x}^{\mathbf{H}}(t)
$$

Conventional beamformer is also known as Delay and Sum method, Fourier method or Bartlett method. It is one of the simplest methods of estimating DOA. At a particular direction $\theta$, the power of the signal is [1]

$$
\overline{\mathbf{P}}_{C B F}(\theta)=\frac{\mathbf{A}^{H}(\theta) \hat{\mathbf{R}}_{\mathbf{x x}} \mathbf{A}(\theta)}{\mathbf{A}^{H}(\theta) \mathbf{A}(\theta)} \text { where, } \theta \text { is from 0-180 degree }
$$

Capon beamformer is formulated according to the Minimum Variance distortionless Response (MVDR) algorithm [2]. It was designed to overcome the limitation (poor resolution problem) of the conventional beamformer. Capon beamformer proposes to minimize the contribution of undesired signals and noises by minimizing the total output power while maintaining the gain along to the look direction constant. Equation for the Capon beamformer is as follows:

$$
\overline{\mathbf{P}}_{\text {Capon }}(\theta)=\frac{1}{\mathbf{A}^{H}(\theta) \hat{\mathbf{R}}_{\mathbf{X X}}^{-1} \mathbf{A}(\theta)}
$$

Multiple Signal Classification (MUSIC) method was first developed by Schmidt [3], Bienvenue and Kopp [4]. It is one of the most popular subspace-based techniques to estimate DOA. It is dependent on the spatial covariance matrix of the data. Assuming $\mathbf{R}_{\mathrm{S}}=\mathrm{E}\left\{\mathbf{s}(\mathrm{t}) \mathbf{s}^{\mathrm{H}}(\mathrm{t})\right\}$ is the source covariance matrix and $\mathrm{E}\left\{\mathbf{n}(\mathrm{t}) \mathbf{n}^{\mathrm{H}}(\mathrm{t})\right\}=\sigma^{2} \mathbf{I}$ is the noise covariance matrix.

So that, $\hat{\mathbf{R}}_{\mathbf{X x}}=\mathbf{A}(\theta) \mathbf{R}_{\mathbf{S}} \mathbf{A}^{\mathrm{H}}(\theta)+\sigma^{2} \mathbf{I}=\mathbf{Q} \mathbf{\Lambda} \mathbf{Q}^{\mathrm{H}}$

$=\mathbf{E}_{\mathrm{S}} \boldsymbol{\Lambda}_{\mathrm{S}} \mathbf{E}_{\mathrm{S}}^{\mathrm{H}}+\mathbf{E}_{\mathrm{N}} \boldsymbol{\Lambda}_{\mathrm{N}} \mathbf{E}_{\mathrm{N}}^{\mathrm{H}}$

where, $\mathbf{Q}$ is a unitary matrix and $\boldsymbol{\Lambda}$ is a diagonal matrix of real eigenvalues such that $\lambda_{1} \geq \lambda_{2} \geq \lambda_{3} \geq \ldots \geq \lambda_{L} . \quad \Lambda \mathrm{s}$ contains the diagonal signal eigenvalues; $\boldsymbol{\Lambda}_{\mathrm{N}}$ contains the diagonal noise eigenvalues. $\mathbf{E}_{S}$ contains eigenvectors associated with the signal eigenvalues (signal subspace matrix). $\quad \mathbf{E}_{\mathrm{N}}$ contains eigenvectors associated with the noise eigenvalues (noise subspace matrix).

Any vector that is orthogonal to $\mathbf{A}(\theta)$ is an eigenvector of $\hat{\mathbf{R}}_{\mathbf{X x}}$ with the eigenvalue $\sigma^{2}$. It is clear that $(L-P)$ such vectors, which are linearly independent. The rest of the eigenvalues are larger than $\sigma^{2}$. So the eigenvalues (and corresponds their eigenvectors) of $\hat{\mathbf{R}}_{\mathbf{X x}}$ can be divided into two groups:

Signal eigenvalues: $\lambda_{i}>\sigma^{2} ; i=1,2,3, \ldots, P$

Noise eigenvalues: $\lambda_{i}=\sigma^{2} ; i=P+1, P+2, P+3, \ldots, L$

Since array steering vectors are orthogonal to the noise subspace eigenvectors at the angles $\theta_{1}, \theta_{2}, \theta_{3}, \ldots, \theta_{P}$ i.e., $\mathbf{A}^{\mathrm{H}}(\theta)$ $\mathbf{E}_{\mathbf{N}}=0$ can show that Euclidean distance $\mathbf{A}^{\mathrm{H}}(\theta) \mathbf{E}_{\mathbf{N}} \mathbf{E}_{\mathbf{N}}^{\mathbf{H}} \mathbf{A}(\theta)=0$ for each and every arrival angel $\theta_{1}, \theta_{2}, \theta_{3}, \ldots, \theta_{P}$.

In practice, a sharp peak is found at an angle of arrival by placing this Euclidean distance in the denominator. Therefore, the DOAs can now be estimated from the $P$ peaks of the following MUSIC function [6]

$$
\overline{\mathbf{P}}_{\text {MUSIC }}(\theta)=\frac{1}{\mathbf{A}^{H}(\theta) \hat{\mathbf{E}}_{N} \hat{\mathbf{E}}_{N}^{H} \mathbf{A}(\theta)}
$$

Estimation of Signal Parameters via Rotational Invariance Technique (ESPRIT) was proposed by Paulraj, Roy and Kailath [7]. It is a search-free DOA estimation technique based on the shift-invariance property of the ULA steering matrix [3]. ESPRIT is considered as a computationally efficient technique compared with MUSIC.

Recall the equation (2) for the steering matrix $\mathbf{A}$ and let $\overline{\mathbf{A}}$ and $\underline{\mathbf{A}}$ be the matrices with eliminated first and last row respectively as shown in Figure 2.

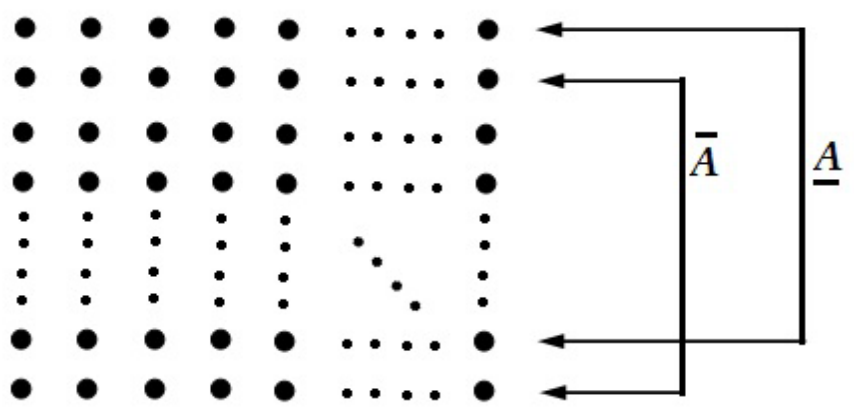

Figure 2: Formation of $\overline{\mathbf{A}}$ and $\underline{\mathbf{A}}$ from $\mathbf{A}$ steering matrix.

Similarly Let $\overline{\mathbf{E}}_{\mathbf{S}}$ and $\underline{\mathbf{E}}_{\mathbf{S}}$ are formed from the signal eigenvector matrix $\mathbf{E}_{\mathrm{S}}$ in the same way as $\overline{\mathbf{A}}$ and $\underline{\mathbf{A}}$ from $\mathbf{A}$. A full rank $P \times P$ matrix $\mathbf{C}$ existed because $\mathbf{E}_{\mathrm{S}}$ and $\mathbf{A}$ span the same subspace namely signal subspace. 
In practice, the sample covariance matrix $\overline{\mathbf{R}}_{X X}$ is used. Then, after computing the signal subspace estimation $\hat{\overline{\mathbf{E}}}_{S}$ the DOAs can be estimated from

$\hat{\theta}_{l}=\cos ^{-1}\left(\frac{-\hat{g}_{l} \times \lambda}{2 \pi d}\right)$

where, $\hat{g}_{l}$ for $l=1,2,3, \ldots, P$ are the eigenvalues of $\hat{\mathbf{\Psi}}=\left(\hat{\overline{\mathbf{E}}}_{\mathbf{S}}^{\mathbf{H}} \hat{\overline{\mathbf{E}}}_{\mathbf{S}}\right)^{-\mathbf{1}} \hat{\overline{\mathbf{E}}}_{\mathbf{S}} \hat{\mathbf{E}}_{\mathbf{S}}$. Here, $\hat{\mathbf{\Psi}}$ is the estimation of $\boldsymbol{\Psi}=\mathbf{C}^{-1} \mathbf{D C}$, where $\mathbf{D}$ is a matrix as

$\mathbf{D}=\left[\begin{array}{cccc}Z_{1} & 0 & \cdots & 0 \\ 0 & Z_{2} & \cdots & 0 \\ \vdots & \vdots & \ddots & \vdots \\ 0 & 0 & \cdots & Z_{L}\end{array}\right]$ where, $Z_{i}=e^{j \mu_{i}}$

\section{A 2D PARAMETER ESTIMATION METHOD}

After exploring the signal model for ULA, now move on to model signal for the URA that involves complex geometries [5] [8]. Figure 3 shows a simple model for the URA, where $K$ and $L$ identical omni-directional sensors along with $x$ - and $y$ axis, respectively. Consider the distance between two adjacent sensors $d x$ along with $x$ - axis and $d y$ along with $y$-axis. It is also considered that $d x=d y=\lambda / 2$. Assuming that $\mathrm{P}$ narrowband coherent radiating sources located in the far field region with wavelength $\lambda$ impinge on the URA at azimuth angle $\theta_{P}$ and elevation $\varphi_{P}$ for $p=1,2,3, \ldots, P$.

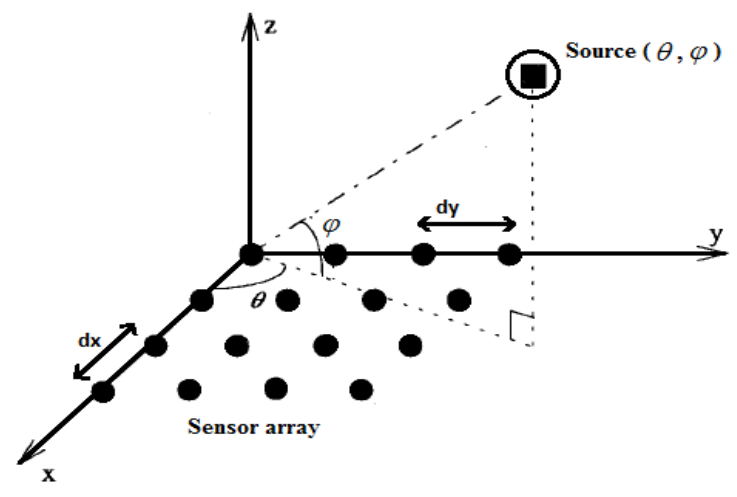

Figure 3: Array geometry of the URA.

Using analytical signal representation [9], received signal at $(i, j)$ th sensor (for $i=1,2,3, \ldots, K$ and $j=1,2,3, \ldots, L$ ) could be mentioned as

$\mathbf{x}(t)=\mathbf{A s}(t)+\mathbf{n}(t)$

here, $\mathbf{n}(t)=\left[n_{1,1}(t), n_{2,1}(t), \ldots, n_{K-1, L}(t), \ldots, n_{K, L}(t)\right]^{\mathrm{T}}$ and $\mathbf{x}$ $(t)=\left[x_{1,1}(t), x_{2,1}(t), \ldots, x_{K-1, L}(t), \ldots, x_{K, L}(t)\right]^{\mathrm{T}}$ and for $t=1$, $2,3, \ldots, T$ times snapshot. Here $[.]^{\mathrm{T}}$ represents the transpose of a matrix $\mathbf{s}(t)=\left[s_{1}(t), s_{2}(t), \ldots, s_{P}(t)\right]^{\mathrm{T}}$ which is $P \times 1$ baseband signal vector and $\mathbf{A}$ is a $K L \times P$ steering matrix whose columns have the form as $\boldsymbol{v e c}\left(\mathbf{a}_{K}(\mu) \mathbf{a}_{L}^{T}(v)\right)$ for $\mu=\mu_{1,} \mu_{2,} \mu_{3, \ldots,} \mu_{P}$ and $v=v_{1}, v_{2}, v_{3}, \ldots, v_{P}$ where, $v_{P}$ is DOA parameters corresponding to the $P$-th source along with $y$-direction and $\mu_{P}$ for $x$-direction.

Here, $\mathbf{a}_{K}(\mu)$ and $\mathbf{a}_{L}(v)$ hold the Conjugate Centro-Symmetric property that denotes steering vectors for URA.

As per equation (3), Covariance matrix for the array output signal $\mathbf{x}(t)$ can be determined. Following equation (6), $\hat{\mathbf{R}}_{\mathrm{Xx}}$ has $K L-P$ eigenvalues equal to the noise power $\sigma^{2}$. Now eigen-decomposition is applied to $\hat{\mathbf{R}} \mathrm{xx}$ in order to obtain the noise and signal subspaces,

$$
\hat{\mathbf{R}}_{\mathrm{Xx}}=\mathbf{E}_{\mathrm{S}} \boldsymbol{\Lambda}_{\mathrm{S}} \mathbf{E}_{\mathrm{S}}^{\mathrm{H}}+\mathbf{E}_{\mathrm{N}} \boldsymbol{\Lambda}_{\mathrm{N}} \mathbf{E}_{\mathrm{N}}^{\mathrm{H}}
$$

here, $(K L-P)$ matrix $\mathbf{E}_{\mathrm{S}}$ contains the eigenvectors corresponding to the signal eigenvalues and $K L \times(K L-P)$ matrix $\mathbf{E}_{\mathrm{N}}$ contains the eigenvectors corresponding to the noise eigenvalues. In (10), $P \times P$ diagonal matrix $\boldsymbol{\Lambda}$ s contains signal eigenvalues and $(K L-P) \times(K L-P)$ diagonal matrix $\Lambda_{\mathbf{N}}$ indicates noise eigenvalues.

Although unitary 2D- and multi-dimensional (MD) ESPRIT [10][11] for the URAs are generalized nowadays, ESPRIT was first developed for 1D DOA estimation in the ULAs.

\section{Multipath CHANNEL ESTIMATION}

Consider $P$ discrete sources of signal and each source has a propagation path to arrive from the transmitter to receiver. It is assumed that some parameters like DOA $(\theta)$, propagation delay $\left(\tau_{P}\right)$ are also involved in each path. In order to fit with standard data transmission, data is first sent through the low pass filter and after that sampling is done at sampling period $T_{S}$.

Assume that the model is 2D. Therefore, an array of receive antennas and two sources are considered. In order to realize the multipath, same signal was transmitted from two different sources but the second one is delayed for a known value. Now, two parameters of interest are DOA, $\theta_{p}$ and propagation delays, $\tau_{P}$ for $p=1,2,3, \ldots, P$. The goal is to estimate $P$ pairs of exponential $\left(a_{p}, b_{p}\right)$ which can be seen as a $2 \mathrm{D}$ harmonic problem [12].

Considering $l=1,2, \ldots, L$ denotes the antenna element index along one dimension of the array output data and $k=1$, $2, \ldots, K$ is the frequency subcarrier index along the other dimension of the array output data.

From [12], equation for the 2D can be formulated as

$$
y_{k, l}(\mathrm{i})=\sum_{p=1}^{P} z_{p}(\mathrm{i}) \mathbf{a}_{p}^{k} \mathbf{b}_{p}^{l}+\mathbf{n}_{k, l}(\mathrm{i})
$$

where, $\quad \mathbf{a}_{p}=e^{-j(2 \pi / \mathrm{K}) \tau_{p}}$

And $\mathbf{b}_{p}=e^{-j\left(2 \pi d_{R} / \lambda\right) \cos \theta_{p}}$

here, $d_{R}$ represents the elemental spacing on the receive side. The steering vector $\mathbf{a}_{p}=\left[a_{p}^{1}, a_{p}^{2}, \ldots, a_{p}^{K}\right]^{T} \quad$ and $\mathbf{b}_{p}=\left[b_{p}^{1}, b_{p}^{2}, \ldots, b_{p}^{L}\right]^{T}$ are the two parameters of interest for $p$-th path. A column of $K \times P$ matrix $\mathbf{A}=\left[\mathbf{a}_{1}, \mathbf{a}_{2}, \ldots, \mathbf{a}_{P}\right]$ could be 
formed from $\mathbf{a}_{P}$. It should be emphasized, although here, it is the same notation for the array steering matrix, namely $\mathbf{A}$, the definition of A is slightly different compared with equation (2) although structures are identical. Similarly vector forms the column of $L \times P$ matrix defined as $\mathbf{B}=\left[\mathbf{b}_{1}, \mathbf{b}_{2}, \ldots, \mathbf{b}_{P}\right]$. Using Khatri-Rao product an equation can be formed as

$\mathbf{B}^{\circ} \mathbf{A}=\left[\mathbf{b}_{1} \otimes \mathbf{a} 1, \mathbf{b}_{2} \otimes \mathbf{a} 2, \ldots, \mathbf{b}_{p} \otimes \mathbf{a} r\right]$

A new vector could be introduced as $\boldsymbol{\Omega}=\left[\left(\mathbf{a}_{\mathbf{1}}, \mathbf{b}_{1}\right), \ldots,\left(\mathbf{a}_{P}, \mathbf{b}_{P}\right)\right]$. Finally, 2D harmonic retrieval problem could be stated as [12] $\mathbf{y}(i)=\mathbf{H}(\boldsymbol{\Omega}) \mathbf{z}(i)+\mathbf{n}(i)$

where, $\mathbf{y}(i)$ indicates the measurement vector and $), \mathbf{z}(i)=[z 1$, $z 1, \ldots, z 1]$ is the complex envelope of the harmonics, which is treated as a nuisance parameter and $\mathbf{n}(i)$ is a vector stands for additive zero-mean complex Gaussian noise with covariance matrix $\sigma^{2} \mathbf{I}_{K L}$.

It is to be mentioned that equation (13) has a similar structure as the signal model that was introduced in equation (9). Comparing with the matrix $\mathbf{A}$ in equation (9), matrix $\mathbf{H}$ has the parameters of interest similar to the structure A. $\mathbf{z}(i)$ also has the similar structure as the complex vector $\mathbf{s}(t)$ in equation (9). Therefore, applying the unitary 2D ESPRIT, it is possible to estimate DOA and path delays from the channel using data vector $\mathbf{y}(i)$ in equation (13). Here, $\mathbf{H}(\Omega)$ is in the frequency domain that corresponds to the required channel.

\section{Simulation RESUlt}

Considering two discrete paths from transmitter to receiver a multipath model is developed. Same signal is transmitted to the both sources via two different antennas whereas second signal is delayed for a small time period. Now it acts as two multipaths from the same source to destination. Basic parameter setups are shown as in Table 1. Table 2 shows the comparison of delays using 2D ESPRIT technique and SCME model for several experimental setups.

Table 1: Simulation parameters setup

\begin{tabular}{|c|c|c|c|}
\hline Attribute & Values & Attribute & Values \\
\hline Sources & 3 & $\begin{array}{c}\text { Carrier } \\
\text { Frequency }\end{array}$ & $2.4 \mathrm{GHz}$ \\
\hline Sensors & 10 & $\begin{array}{c}\text { Distance } \\
\text { between two } \\
\text { adjacent } \\
\text { antennas }\end{array}$ & $0.0625 \mathrm{~m}$ \\
\cline { 1 - 2 } $\begin{array}{c}\text { Time } \\
\text { samples }\end{array}$ & 100 & Noise Variance & 2 \\
\hline Power (w) & {$\left[\begin{array}{lll}1 & 1 & 1\end{array}\right]$} & \\
\cline { 1 - 2 } True Angel & {$\left[\begin{array}{lll}50 & 60 & 110\end{array}\right]$} & & \\
\hline
\end{tabular}

In the simulation, sensor spacing is usually half of the wavelength. Larger spacing results in performance degradation. Spatial resolution directly depends on the number of sensors, i.e., if the numbers of sensors with proper spacing are increased spatial resolution is also improved. If the sources are closely spaced, it is not possible to distinguish the actual direction of arrival. It is seen that in Figure 4, Capon beamformer just gets the better of this problem. Apart from this, MUSIC shows a high-resolution property. But to get the better result, the number of sources must be smaller than the total number of sensors in the array. For low SNR conditions and closely spaced signals, MUSIC is not able to perform well. Accuracy also depends on the number of snapshots. Multipath is a major problem since MUSIC is unable to estimate DOAs of the correlated signals correctly. From the simulation, ESPRIT technique performs the best among. However, it is not plotted on the graph because it is a search-free estimation technique.

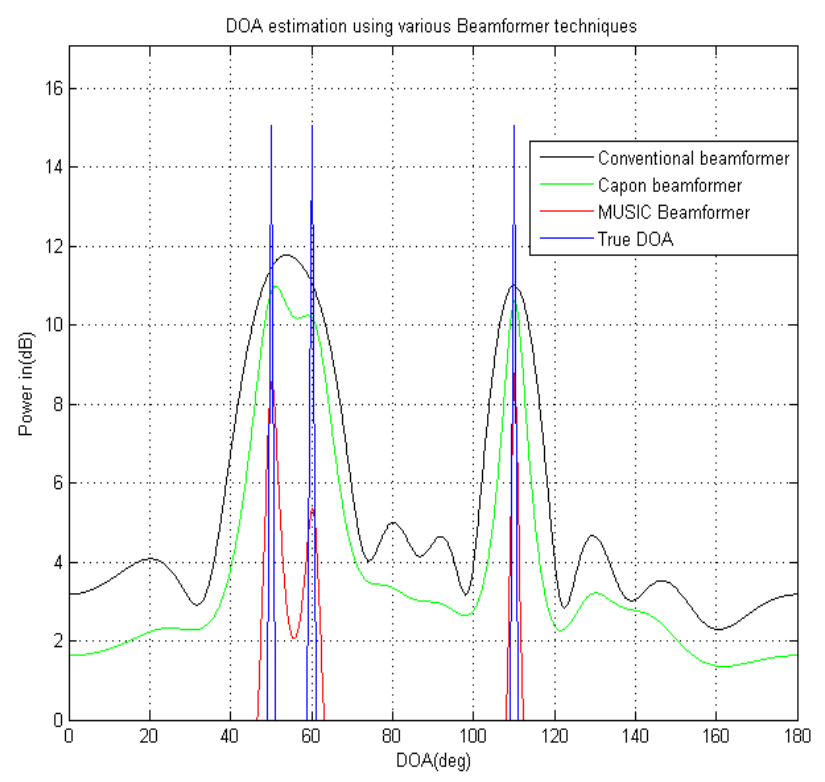

Figure 4: DOA estimation using various techniques

Table 2: Comparison of direction of arrival and multipath delay estimation using 2D ESPRIT technique and SCME model between true and estimated data.

\begin{tabular}{|c|c|c|}
\hline Text & True & Estimated \\
\hline $\begin{array}{l}\text { Direction } \\
\text { Arrival (1) }\end{array}$ & [20 60] Degree & $\begin{array}{ll}{[20.156} & 60.001] \\
\text { Degree } & \end{array}$ \\
\hline Delay (second)(1) & $\begin{array}{l}{[1.0 \mathrm{e}-06 \quad * \quad 0} \\
1.0 \mathrm{e}-06 * 0.2604]\end{array}$ & $\begin{array}{l}{[1.0 \mathrm{e}-06 * 0} \\
06 * 0.2644]\end{array}$ \\
\hline $\begin{array}{l}\text { Direction } \\
\text { Arrival (2) }\end{array}$ & [60 120] Degree & $\begin{array}{ll}60.602 & 120.221] \\
\text { Degree } & \end{array}$ \\
\hline Delay(second) (2) & $\begin{array}{l}{[1.0 \mathrm{e}-06 \quad * \quad 0} \\
1.0 \mathrm{e}-06 * 0.2604]\end{array}$ & $\begin{array}{ll}{[1.0 \mathrm{e}-06 * 0} & 1.0 \mathrm{e}- \\
06 * 0.2504] & \end{array}$ \\
\hline $\begin{array}{l}\text { Direction } \\
\text { Arrival (3) }\end{array}$ & [30 90] Degree & $\begin{array}{ll}30.156 & 90.031] \\
\text { Degree } & \end{array}$ \\
\hline Delay(second) (3) & $\begin{array}{l}{[1.0 \mathrm{e}-06 \quad * \quad 0} \\
1.0 \mathrm{e}-06 * 0.2604]\end{array}$ & $\begin{array}{l}{[1.0 \mathrm{e}-06 * 0} \\
06 * 0.2515]\end{array}$ \\
\hline
\end{tabular}

\section{CONCLUSIONS}

In this paper, a 2D channel estimation technique by using LTE reference signals and SCME model has been considered and simulated. Starting from basic DOA estimation techniques, we moved on to estimate the channel. To make sure that the estimated channel was correct, we also estimated DOA and delays. For this, it was required to consider a single source with multipath propagation model. We followed the LTE 
standard provided by the 3GPP in order to find the channel. The results were compared with known parameters and found almost similar results.

Since, only two paths were considered for estimating the channel, the work might be extended by considering more paths. By extending this model, it is possible to explore a new era of communication systems.

\section{REFERENCES}

[1] Krim, H., \& Viberg, M. (1996). Two decades of array signal processing research: the parametric approach. Signal Processing Magazine, IEEE, 13(4), 67-94

[2] Capon, J. (1969). High-resolution frequency-wavenumber spectrum analysis.Proceedings of the IEEE, 57(8), 1408-1418.

[3] Schmidt, Ralph O. "Multiple emitter location and signal parameter estimation."Antennas and Propagation, IEEE Transactions on 34, no. 3 (1986): 276-280.

[4] Bienvenu, G., \& Kopp, L. (1983). Optimality of high resolution array processing using the eigensystem approach. Acoustics, Speech and Signal Processing, IEEE Transactions on, 31(5), $1235-1248$.

[5] Ullah, H. Multi-antenna aided positioning in lte networks," Master's thesis, Technische Universitaet Darmstadt, 2013 available at Ullah, H. Multi-antenna aided positioning in lte networks," Master's thesis, Technische Universitaet Darmstadt, 2013 available at www.academia.edu/4256293/Theis_report.
[6] Khallaayoun, A. (2010). High resolution direction of arrival estimation analysis and implementation in a smart antenna system (Doctoral dissertation, Montana State UniversityBozeman, College of Engineering).

[7] Paulraj, A., Roy, R., \& Kailath, T. (1986). A subspace rotation approach to signal parameter estimation. Proceedings of the IEEE, 74(7), 1044-1046.

[8] Ren S, Ma X, Yan S, Hao C. 2-D Unitary ESPRIT-Like Direction-of-Arrival (DOA) Estimation for Coherent Signals with a Uniform Rectangular Array.Sensors. 2013; 13(4):42724288.

[9] Shan, T. J., Wax, M., \& Kailath, T. (1985). On spatial smoothing for direction-of-arrival estimation of coherent signals. IEEE Transactions on Acoustics, Speech, and Signal Processing, 33(4), 806-811.

[10] Haardt, M., \& Nossek, J. A. (1995). Unitary ESPRIT: How to obtain increased estimation accuracy with a reduced computational burden. Signal Processing, IEEE Transactions on, 43(5), 1232-1242.

[11] Zoltowski, M. D., Haardt, M., \& Mathews, C. P. (1996). Closed-form 2$\mathrm{D}$ angle estimation with rectangular arrays in element space or beamspace via unitary ESPRIT. Signal Processing, IEEE Transactions on, 44(2), 316-328.

[12] Pesavento, M., Mecklenbräuker, C. F., \& Böhme, J. F. (2004). Multidimensional rank reduction estimator for parametric MIMO channel models. EURASIP Journal on Applied Signal Processing, 2004, 1354-1363. 